\title{
Short, large-amplitude speed enhancements in the near-Sun fast solar wind
}

\author{
T. S. Horbury, ${ }^{\star}$ L. Matteini† and D. Stansby \\ The Blackett Laboratory, Imperial College London, Exhibition Road, London SW7 2AZ, UK
}

Accepted 2018 April 11. Received 2018 April 10; in original form 2018 January 23

\begin{abstract}
We report the presence of intermittent, short discrete enhancements in plasma speed in the nearSun high-speed solar wind. Lasting tens of seconds to minutes in spacecraft measurements at $0.3 \mathrm{au}$, speeds inside these enhancements can reach $1000 \mathrm{~km} \mathrm{~s}^{-1}$, corresponding to a kinetic energy up to twice that of the bulk high-speed solar wind. These events, which occur around 5 per cent of the time, are Alfvénic in nature with large magnetic field deflections and are the same temperature as the surrounding plasma, in contrast to the bulk fast wind which has a wellestablished positive speed-temperature correlation. The origin of these speed enhancements is unclear but they may be signatures of discrete jets associated with transient events in the chromosphere or corona. Such large short velocity changes represent a measurement and analysis challenge for the upcoming Parker Solar Probe and Solar Orbiter missions.
\end{abstract}

Key words: Sun: corona-solar wind.

\section{INTRODUCTION}

The solar wind is a fast, continuous plasma outflow from the Sun's hot corona which fills interplanetary space. It is composed, broadly, of two types: a mostly fast $\left(\approx 700 \mathrm{~km} \mathrm{~s}^{-1}\right)$ flow from magnetically open 'coronal holes' and a more variable, slower $(\approx 250$ $500 \mathrm{~km} \mathrm{~s}^{-1}$ ) wind, whose precise origin is less clear and may be transient in nature. High-speed streams are typically rather homogeneous on scales of hours to days, in both speed and density.

The coronal source of the fast solar wind, however, is anything but steady. Variations have traditionally been classified as either waves or transients. The corona is pervaded by wave motions, probably largely Alfvénic in nature. Apparent preferential heavy ion heating (Cranmer 2002) suggests that significant wave-particle energy transfer plays a role in energizing the plasma. In situ measurements of the fast wind show ubiquitous large-amplitude anti-Sunward propagating Alfvénic fluctuations (Bruno \& Carbone 2013) with a $f^{-1}$ power spectrum at large scales and an active $f^{-5 / 3}$ turbulent cascade at smaller scales. The $f^{-1}$ fluctuations reduce in amplitude with distance in agreement with the WKB approximation, implying that they are not undergoing an active energy cascade or significant damping (Bavassano et al. 1982; Horbury \& Balogh 2001). These fluctuations must originate near the Sun, either as the remnants of waves in the corona or from some other process.

\footnotetext{
^E-mail: t.horbury@imperial.ac.uk

$\dagger$ Present address: LESIA, Observatoire de Paris, Université PSL, CNRS, Sorbonne Université, Univ. Paris Diderot, Sorbonne Paris Cité, 5 place Jules Janssen, F-92195 Meudon, France.
}

In addition to waves, the corona, photosphere, and chromosphere host a wide array of energetic transient phenomena including plumes, spicules, and coronal hole jets (Raouafi et al. 2016). While plumes are large and long-lasting, the increasing temporal and spatial resolution of spacecraft observations has led to the discovery of progressively smaller and faster events, such as IRIS observations of hot jets within coronal holes on time-scales of around a minute (Tian et al. 2014).

The contribution of solar transients to the energy and mass flux of the solar wind remains unclear. Attempts have previously been made to link speed variations in fast wind, on spacecraft scales of hours, to supergranule structure (Thieme, Schwenn \& Marsch 1989). Plumes in coronal holes are thought to be related to solar wind microstreams in the high-speed polar solar wind at several Astronomical Units (Neugebauer 2012), with speed variations of $\approx 35 \mathrm{~km} \mathrm{~s}^{-1}$ on spacecraft scales of hours but smaller scale structures might not be expected to survive out to the large solar distances $(>60$ solar radii, $R_{\mathrm{S}}$ ) at which we currently have in situ measurements. Remote measurements of such events in the solar atmosphere cannot on their own demonstrate the events' contribution to the overall solar wind flow, although coronagraph observations of polar fast wind streams (Jackson et al. 2014) show transient speed variations of hundreds of $\mathrm{km} \mathrm{s}^{-1}$ at a few $R_{\mathrm{S}}$. Simulations suggest that transient events can contribute to the solar wind energy budget (e.g. Cranmer \& Woolsey 2015) but to date, there has not been good evidence of the remnants of such impulsive events in the solar wind itself.

More recently, observations of spicules and coronal jets have motivated simulations of these events (Karpen et al. 2017; Roberts et al. 2017; Uritsky et al. 2017; Wyper, Antiochos \& DeVore 2017). 
These simulations, with rather different geometries and triggering mechanisms, nevertheless generate qualitatively similar signatures: a hot plasma jet and an upwardly propagating Alfvénic disturbance. While the simulated jets interact with the ambient plasma and hence do not propagate to large distances, the Alfvénic disturbances, being essentially undamped and travelling at the Alfvén speed, can travel rapidly through the background plasma and far from the Sun (Uritsky et al. 2017), up to at least $10 R_{\mathrm{S}}$ (Roberts et al. 2017).

Recently, Matteini et al. $(2014,2015)$ have pointed out that the mean (Parker spiral) magnetic field direction, combined with the near-constant magnetic field magnitude in the presence of antiSunward propagating Alfvénic fluctuations, results in an asymmetry in the variations in the solar wind speed, with large enhancements being more common than reductions, first observationally identified by Gosling, Tian \& Phan (2011). This geometrical effect is more pronounced at lower Parker spiral angles and since the amplitude of the speed variations can in principle reach twice the Alfvén speed for large-amplitude fluctuations, the amplitudes of these events could be larger at lower solar distances for a given field angle change. This asymmetry in speed variations is present in the solar wind at $60 R_{\mathrm{S}}$ (Matteini et al. 2015) but by itself does not demonstrate a particular origin of the Alfvénic fluctuations themselves.

In this work, we consider these velocity variations in more detail and demonstrate that the near-Sun fast solar wind is not smooth, but is filled with short Alfvénic velocity spikes up to $250 \mathrm{~km} \mathrm{~s}^{-1}$ above the background wind, which carry a small but significant fraction of the total kinetic energy of the plasma. These spikes last tens of seconds to minutes and recur on scales of minutes to tens of minutes, and might therefore be the direct signatures of impulsive chromospheric or coronal energy release. If true, this potential link would open a new window on impulsive solar processes, which are a ubiquitous aspect of solar dynamics.

We present examples of these events and evidence for their ultimate origins. In addition since the velocity variations are so large and can occur on time-scales of seconds, we discuss the resulting challenges for measurements of the solar wind in the inner heliosphere by upcoming missions, along with how such variations can be allowed for when studying solar wind structure and small-scale turbulence.

\section{OBSERVATIONS OF VELOCITY SPIKES}

The closest in situ solar wind measurements to the Sun to date were taken by the twin Helios probes at $0.29 \mathrm{au}\left(43 \times 10^{6} \mathrm{~km}\right.$ or $62 R_{\mathrm{S}}$ ). Fig. 1 shows several days of the bulk plasma speed during one high-speed stream measured by Helios 2 at 0.29 au in 1976. Also shown are two intervals, of the same length, in high-speed streams at 1 au at low latitudes (from Wind in 2008) and 2.4 au at high latitudes (from Ulysses in 1995). While the polar stream is generally smoother and all three exhibit variations on time-scales of several hours associated with microstreams (Neugebauer 2012), the most striking difference between the three data sets is the large, short speed enhancements at 0.3 au throughout the interval.

A shorter interval of 0.3 au data (Fig. 2) shows the broadly homogeneous stream, with a near-constant magnetic field magnitude, density, and temperature. In contrast, the velocity is spiky and in particular the spikes are clearly asymmetric, appearing as enhancements over a slower background. These spikes are generally anticorrelated with variations in the radial magnetic field component, consistent with them being Alfvénic fluctuations with an anti-Sunward propagation sense in the solar wind frame (e.g. Matteini et al. 2014). Indeed, there is a field-velocity anticorrelation in all three compo-

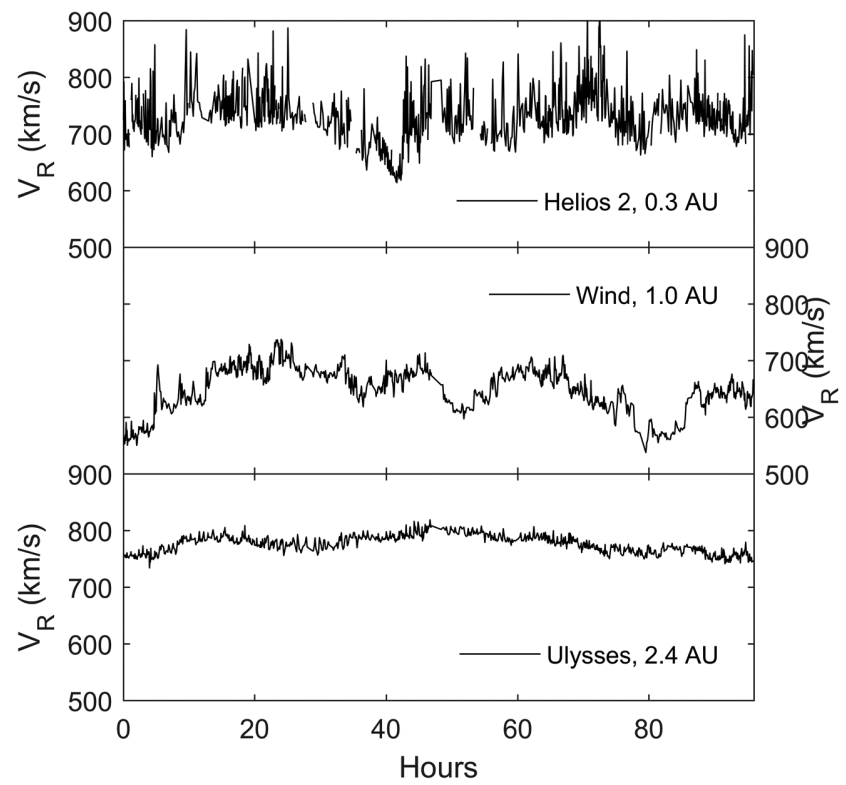

Figure 1. Radial solar wind speed in three different high-speed streams, at 0.3 (Helios 2, starting at $1976 \mathrm{~d} \mathrm{106)}, 1.0$ (Wind, $2008 \mathrm{~d} 18$ ), and 2.4 au (Ulysses, 1995 d 268) from the Sun. The Helios (40s) and Wind (97s) data have been decimated to the same 4 min cadence as Ulysses to ease comparison.

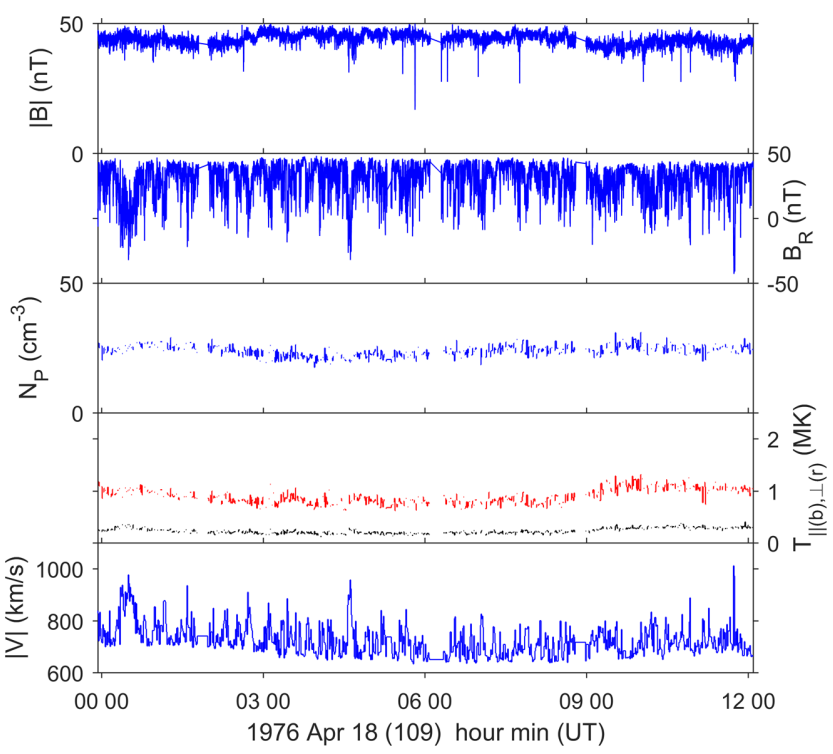

Figure 2. Several hours of a 0.3 au high-speed stream. Top to bottom, panels are: field magnitude, radial field component, proton core number density, core perpendicular (red) and parallel (black) temperatures, and core speed.

nents during this time. Fluctuations on these scales have been extensively studied statistically, both at 0.3 au with Helios and elsewhere (Bruno \& Carbone 2013). What is striking here is that the variations are asymmetric, being dominated by speed increases above a background, and that these enhancements are short and isolated. We proceed to analyse these events in more detail, which requires a consideration of the measurements made by the Helios spacecraft. 


\subsection{Data sets}

In the analysis presented here, we use 4 samples s ${ }^{-1}$ magnetometer data (Musmann et al. 1975) and 40.5 s ion data (Schwenn, Rosenbauer \& Miggenrieder 1975). The measurements are over $40 \mathrm{yr}$ old and present some analysis challenges so care must be taken. The magnetometer data calibration is adequate for our purposes here with an important caveat that values above $50 \mathrm{nT}$ in any component are not present; this does not significantly affect the results here. The plasma data present more challenges; see Marsch et al. (1982) for more detailed discussion.

We timestamp the plasma data based on the time at which the energy of the peak of the distribution function is measured; for highspeed wind, this is around $11 \mathrm{~s}$ after the time of the beginning of the distribution measurement. We have used field-velocity crosscorrelations, taking advantage of the Alfvénicity of the wind, to confirm this time which is important when we are trying to find distributions which have been measured entirely within these short events.

We compute field-parallel and perpendicular temperatures by fitting a bi-Maxwellian to the proton core rather than taking moments, so the supra-thermal proton beam and alpha particles do not affect the values; we crudely estimate the beam fraction as the remainder of the measured distribution. An important limitation is the low cadence of the plasma data. Distributions were built up over multiple spacecraft spins, each of which lasted $1 \mathrm{~s}$. In general, the bulk velocity (from which we remove the spacecraft aberration velocity, about $70 \mathrm{~km} \mathrm{~s}^{-1}$ at $0.29 \mathrm{au}$ ) remains reliable, but core proton temperatures and densities do not. In order to avoid the effects of mixing different particle distributions, we use these temperatures and densities only when the magnetic field remains within $20^{\circ}$ of the mean over the time taken to measure the proton core distribution, which is around $10 \mathrm{~s}$ : this is a significant limitation when the field changes rapidly within these short events. Extensive testing and comparison with moments derived using other algorithms make us confident that the velocities, temperatures, and densities presented here are reliable; the statistical plasma properties presented later in this paper use only these values.

We concentrate here on the high-speed stream measured at 0.29 au by Helios 2 on 1976 d 106 00:00-110 00:00, but similar events are present in other high-speed streams.

\subsection{Example event: 11:44, 1976 April 18}

One large-amplitude, short-velocity enhancement is shown in Fig. 3. This is a particularly clear example, but there are many other similar events. The magnetic field magnitude remained relatively constant throughout the event, which is visible as a reversal in the radial component of the magnetic field. In this example, the magnetic field rotated almost $180^{\circ}$, but this is not the case in all events. The field returned to the ambient near-Parker spiral orientation after around $2 \mathrm{~min}$ and had a sharp boundary at both beginning and end.

Given the short duration of the event, there are very few plasma measurements within and around it - and rapid changes in field direction mean that the proton core temperature and density are unreliable and discarded. Nevertheless, it is clear that the proton radial speed increased to around $1000 \mathrm{~km} \mathrm{~s}^{-1}$ from a background of around $700 \mathrm{~km} \mathrm{~s}^{-1}$; the core protons within the event therefore had around twice the kinetic energy of the surrounding material. Such large-amplitude, short-duration velocity changes do not occur in the high-speed wind at 1 au but are common in the Helios measurements of such wind at $0.29 \mathrm{au}$.

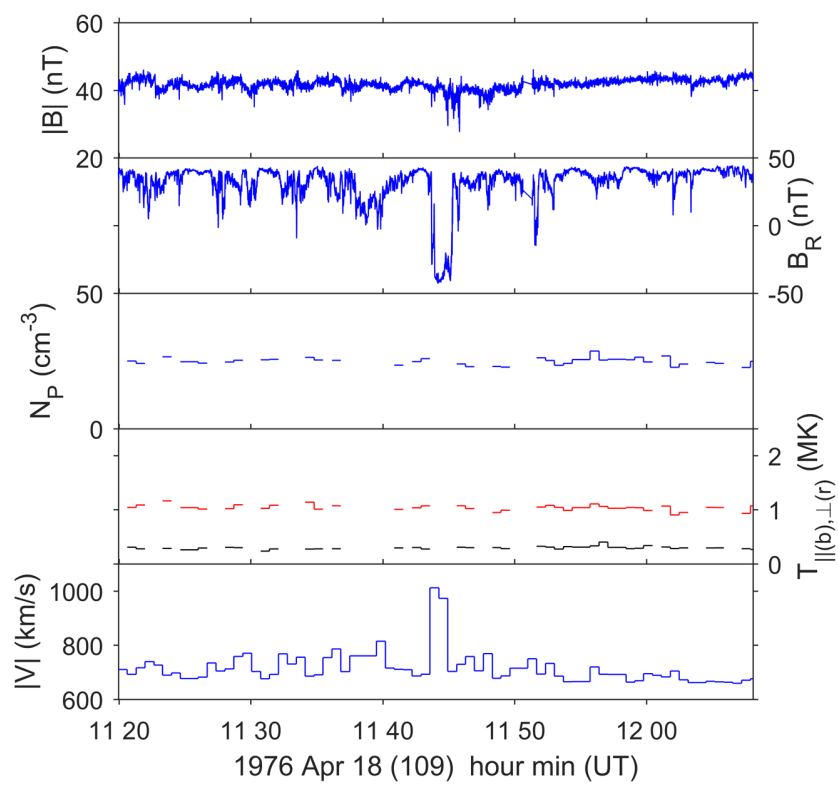

Figure 3. A short duration velocity enhancement observed by Helios 2 in a high-speed solar wind stream at 0.29 au. Panels are, top to bottom, magnetic field magnitude, radial magnetic field component, proton number density, parallel (black) and perpendicular (red) core proton temperature, and core proton speed.

We note that the velocity and magnetic field variations in this event, as is generally true in fast wind, were correlated as for an antiSunward propagating Alfvénic fluctuation. The ion distributions within the event (not shown here) indicate the presence of a proton beam travelling Sunward in the bulk plasma frame, consistent with this being a local 'fold' in the magnetic field, and not a mini-sector of opposite polarity magnetic field.

\subsection{Statistical properties}

While short, sharp speed increases as shown in Fig. 3 are common in the near-Sun fast wind, they vary considerably in duration and amplitude. We have attempted to find them by first calculating a running $30 \mathrm{~min}$ boxcar moving average solar wind speed $V_{\text {smooth }}$ and then $\delta V=V_{\mathrm{SW}}-V_{\text {smooth }}$, the instantaneous deviation from this background (Fig. 4). We identify a velocity spike as a time when $|\delta V|>75 \mathrm{~km} \mathrm{~s}^{-1}$, this rather subjective boundary being around half the local Alfvén speed. Over the $4 \mathrm{~d}$ interval we identified 294 positive speed increases and only 26 speed decreases by the same criterion. Most (221) of the events were only one plasma data point $(40.5 \mathrm{~s})$ in duration, only seven were longer than two data points and the longest was $8 \mathrm{~min}$.

The mean time between events was 19 min but their spacing was rather variable. Their overall amplitude and occurrence rate appear to vary with speed within the fast stream, as can be seen in Fig. 1, with fewer and smaller events when the wind was slower.

The mean speed of the events was $849 \mathrm{~km} \mathrm{~s}^{-1}$, compared to $725 \mathrm{~km} \mathrm{~s}^{-1}$ for the entire interval. The kinetic energy of the plasma within the events was 35 percent higher than the average over the entire stream: the events occurred during 5.4 per cent of its duration, contained 7.2 per cent of the total kinetic energy and 6.2 per cent of the mass flux. Due to the low cadence of the plasma data, however, these figures are likely to be underestimates and of course are dependent on the rather arbitrary threshold of $75 \mathrm{~km} \mathrm{~s}^{-1}$. 


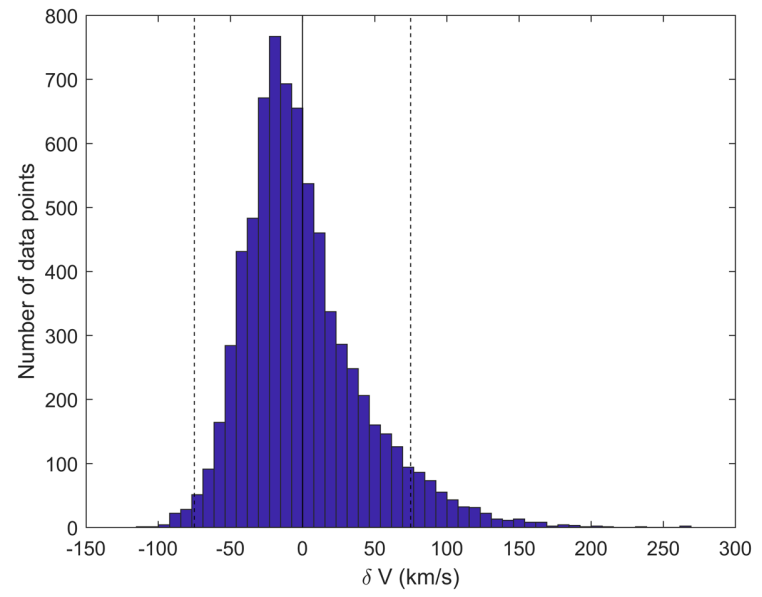

Figure 4. Distribution of radial speeds with respect to a 30 min running mean during a $4 \mathrm{~d}$ high-speed stream at $0.29 \mathrm{au}$.

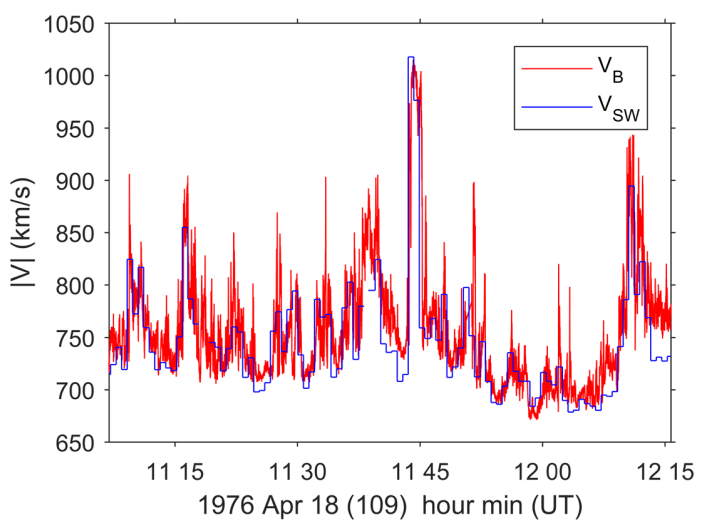

Figure 5. Measured plasma speed ( $V_{\mathrm{SW}}$, blue) and estimated speed using magnetic field data $\left(V_{B}\right.$, red).

We have identified velocity enhancements as being at least $75 \mathrm{~km} \mathrm{~s}^{-1}$ above the $30 \mathrm{~min}$ smoothed profile. Most of these enhancements lasted only one plasma data point. There might therefore be events shorter than the plasma measurement cadence of $40.5 \mathrm{~s}$ which are not resolved. In order to estimate the velocity profile at smaller scales, we take advantage of the high correlation of the Alfvénic velocity and magnetic field fluctuations (Bruno \& Carbone 2013). We can estimate the velocity fluctuations as $\delta \boldsymbol{V}=\alpha \delta \boldsymbol{B}$. In theory, $\alpha=r_{\mathrm{A}} V_{\mathrm{A}} / B_{0}$, where $r_{\mathrm{A}}$ is the Alfvén ratio, $V_{\mathrm{A}}$ the Alfvén speed, and $B_{0}$ the field magnitude. In practice, we estimate $\alpha$ from the $\delta \boldsymbol{V}-\delta \boldsymbol{B}$ correlation at $40.5 \mathrm{~s}$ scale. We then use the 4 samples $\mathrm{s}^{-1}$ field data, and the smoothed background velocity, to estimate the velocity profile as $\boldsymbol{V}_{B}=\boldsymbol{V}_{\text {smooth }}+\alpha \delta \boldsymbol{B}$. An example interval is shown in Fig. 5 where the speed profile estimated from the field data shows considerable substructure compared to that from the plasma data themselves and the maximum amplitudes of the peaks are underestimated by the plasma data. Individual short spikes, unresolved by the plasma data, are also present.

This crude procedure of estimating the velocity variations assumes that at scales below $40.5 \mathrm{~s}$ and up to the magnetic field cadence of 4 vectors $\mathrm{s}^{-1}$, the fluctuations are purely Alfvénic. The proton gyro-scale is typically around $1 \mathrm{~Hz}$ or slightly higher at 0.3 au (Trenchi, Bruno \& Trenchi 2014), below which fluctuations are certainly not Alfvénic, so the estimated velocity profile shown here should be considered with caution, but it is likely to provide a
Table 1. Mean properties inside spikes compared to the entire stream. Error values are standard deviations.

\begin{tabular}{lcc}
\hline & Spikes & Stream \\
\hline Proton core speed $\left(\mathrm{km} \mathrm{s}^{-1}\right)$ & $852 \pm 43$ & $724 \pm 54$ \\
Proton core number density $\left(\mathrm{cm}^{-3}\right)$ & $25.1 \pm 3.2$ & $25.5 \pm 2.8$ \\
Proton core $T_{\|}\left(10^{5} \mathrm{~K}\right)$ & $2.6 \pm 0.6$ & $2.5 \pm 0.5$ \\
Proton core $T_{\perp}\left(10^{5} \mathrm{~K}\right)$ & $9.6 \pm 1.4$ & $9.5 \pm 1.3$ \\
Proton beam fraction & $0.14 \pm 0.04$ & $0.14 \pm 0.04$ \\
Magnetic field magnitude $(\mathrm{nT})$ & $42 \pm 3$ & $43 \pm 3$ \\
\hline
\end{tabular}

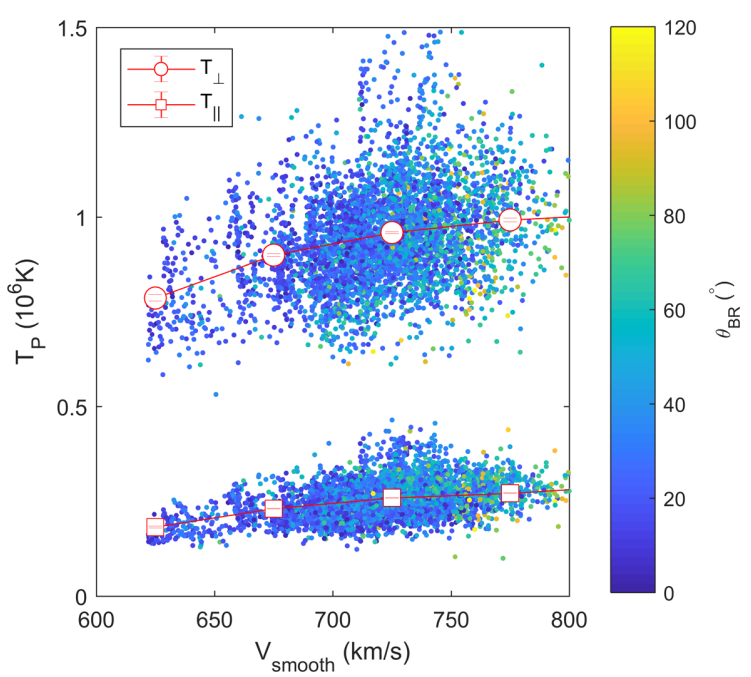

Figure 6. Proton core perpendicular (top) and parallel (bottom) temperatures within a high-speed stream as a function of the 30-min smoothed solar wind speed. Colours of the dots represent angle of the local magnetic field to the radial. Red open circles and squares are means (with standard errors) over bins of $50 \mathrm{~km} \mathrm{~s}^{-1}$.

reasonably representative estimate of the velocity profile at least in a statistical sense. It seems likely that there are substantial numbers of large-amplitude velocity variations even on scales of seconds at $0.3 \mathrm{au}$ in fast wind. Using our earlier criterion of a $75 \mathrm{~km} \mathrm{~s}^{-1}$ speed enhancement and identifying the end of an event when the enhancement reduced below $50 \mathrm{~km} \mathrm{~s}^{-1}$, we find 4032 events covering 10 per cent of the $4 \mathrm{~d}$ interval with a mean time between events of $86 \mathrm{~s}$. These numbers might be overestimates but it is clear from the timeseries that the high-speed solar wind is filled with such structures.

If the events considered here were caused by transient energy release near the Sun, such as from coronal jets, then one might expect the plasma within them to differ from that in the surrounding solar wind. We have found no such difference, for the proton core parallel or perpendicular temperatures, total density, proton beam fraction, or indeed the field magnitude: see Table 1 - note that we have quoted standard deviations in these quantities rather than standard errors in order to illustrate their typical range of variation. It is well known, however, that the proton temperature is correlated with solar wind speed, at least on a large scale. This is also true within the individual fast stream considered here. Fig. 6 shows the proton parallel and perpendicular temperatures plotted against $V_{\text {smooth }}$. This process effectively removes speed variations due to the velocity spikes considered here and recovers the background wind speed, which does not exceed $800 \mathrm{~km} \mathrm{~s}^{-1}$. Although there is considerable scatter in Fig. 6, the dependence of temperature 


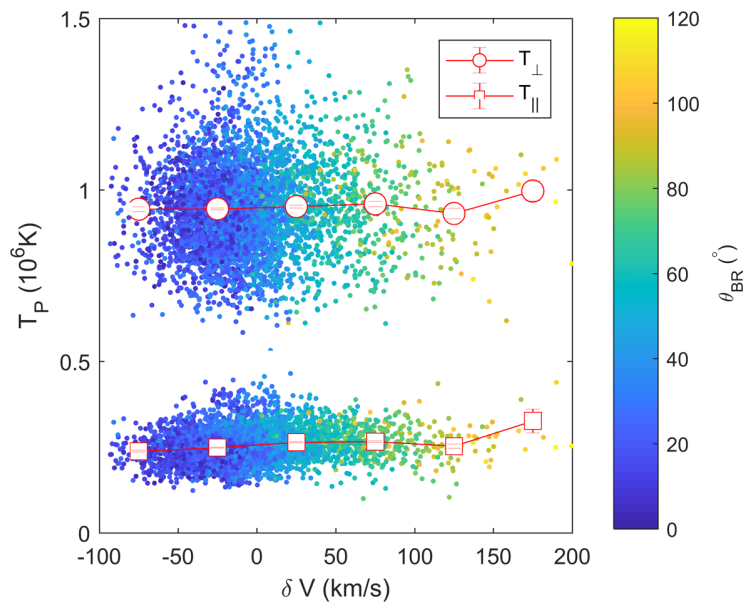

Figure 7. Proton core temperatures in the same format as Fig. 6 but plotted against the local variation from the $30 \mathrm{~min}$ smoothed solar wind speed.

on speed is clear. This variation is quantitatively similar to that previously reported statistically over many streams both at 1 and $0.3 \mathrm{au}$ (Elliott et al. 2012; Hellinger \& Travnicek 2014) but is to our knowledge the first such correlation shown within one solar wind stream. Dots in Fig. 6 are coloured by $\theta_{\mathrm{BR}}$, the angle between the local magnetic field and the radial direction: there is no clear dependence of smoothed speed on magnetic field direction at this large scale, as expected.

In sharp contrast to the meso-scale solar wind variations in Fig. 6, there is no dependence of speed on temperature within the events themselves. This is shown in Fig. 7 where temperatures are plotted against $\delta V$. We can see the clear dependence of $\delta V$ on $\theta_{\mathrm{BR}}$ on these scales, as previously reported by Matteini et al. (2014), again consistent with these being highly Alfvénic fluctuations. A dependence of temperature on $\theta_{\mathrm{BR}}$ would be clear proof that these variations are not simply Alfvénic waves. Lack of such a dependence is not in itself proof of a wave origin however, as we discuss in the next section.

\section{DISCUSSION}

We have presented evidence that the high-speed solar wind at $60 R_{\mathrm{S}}$ contains large numbers of discrete velocity enhancements, lasting from seconds to a few minutes. These events are Alfvénic with an anti-Sunward propagation sense in the solar wind frame and the core proton distribution within them is no different to the ambient wind - these are, therefore, simply Alfvénic fluctuations. The question naturally arises as to their origin.

\subsection{Relation to waves and turbulence}

The events described here occur on time-scales of seconds to minutes, have a broad range of amplitudes, and occur apparently randomly with a mean time between events of $\approx 19 \mathrm{~min}$. Power spectra of fluctuations on these scales at 0.3 au show a $f^{-1}$ spectrum from low frequencies up to around $100 \mathrm{~s}$, above which is turbulence with a $f^{-5 / 3}$ spectrum. The $f^{-1}$ Alfvénic fluctuations in fast wind streams have previously been linked to wave motion in the corona (Bruno, Bavassano \& Villante 1985) - see e.g. Cranmer, Gibson \& Riley (2017), Bruno \& Carbone (2013) for reviews - or a superposition of uncorrelated turbulence samples (Matthaeus \& Goldstein 1986): their decrease in power with distance is consistent with adiabatic expansion with no damping, while the $f^{-5 / 3}$ fluctuations decay more rapidly, consistent with an active turbulent cascade. Alfvénic turbulence is intermittent (e.g. Bruno \& Carbone 2013) and hence it is possible that the velocity spikes are the extreme end of an intermittent distribution caused by an active turbulent cascade. Some of the events, however, have durations beyond the large-scale end of the turbulent cascade (at $\approx 100 \mathrm{~s}$ ) and moreover fluctuations in fast streams are less intermittent closer to the Sun (Bruno et al. 2003) which argues against this interpretation. The lack of an identifiable scale or amplitude means that if these are discrete events, it is not clear what fraction of the total fluctuation power they contain, but they seem not to be the dominant source of power on spacecraft scales of minutes.

Regardless of the origin of the velocity spikes, they appear to evolve with distance as part of the bulk fluctuation population, and their amplitude decreases with solar distance. Since they are large amplitude, $\delta \boldsymbol{B} /|B| \approx 1$ fluctuations, their amplitude is around the Alfvén speed: by 1 au their amplitude is therefore around 40$50 \mathrm{~km} \mathrm{~s}^{-1}$ (Gosling et al. 2011; Matteini et al. 2014), and beyond it is even smaller.

\subsection{Relation to solar transients}

While solar transients have long been observed remotely, one would perhaps not expect events lasting only a few minutes to persist out to $60 R_{\mathrm{S}}$, over a traveltime of nearly a day. Simulations by Roberts et al. (2017) suggest that this might indeed be possible, however, at least to $10 R_{\mathrm{S}}$, and hence perhaps to $60 R_{\mathrm{S}}$. In essence, a reconnection-driven outflow in the corona results in both a plasma jet and an Alfvénic fluctuation propagating anti-Sunward. While the plasma outflow of a few hundred $\mathrm{km} \mathrm{s}^{-1}$ merges back into the ambient medium relatively rapidly, the high Alfvén speed in the lower corona causes the upward-propagating Alfvénic fluctuation to outrun the exhaust. Simulations show this fluctuation developing fine scale substructure as it propagates (Uritsky et al. 2017), but persisting as a discrete structure, with amplitudes near the Alfvén speed and hence resulting in speed enhancements of several hundred $\mathrm{km} \mathrm{s}^{-1}$, at least up to $10 R_{\mathrm{S}}$. We suggest that the events reported here might be precisely these Alfvénic outflows and hence be the in situ signatures of coronal jets.

If the velocity spikes are the signatures of coronal jets, this opens up the prospect of using in situ measurements as another tool to study solar dynamics. For example, the amplitudes of the spikes appear to depend on the underlying wind speed, even within a single stream. This can be seen in Figs 1 and 2, where spikes are smaller when the background wind is slower; Fig. 8 makes this clear statistically. Given the lack of solar observations in 1976, it is not possible to determine whether the background speed variations were due to Helios 2 passing close to the edge of the coronal hole flow or moving through different regions deep within that flow that is, whether both speed and spike amplitude vary even within a fast stream, due to variations in the source region. If further observations show that the latter is the case, this would suggest a close link between impulsive energy input via jets and the bulk speed and temperature of the wind. Indeed, spikes also appear to be present in some slower, but still Alfvénic, wind near 0.3 au.

In situ data also allow an independent estimate of the occurrence rate of these transient events. While we have used a rather arbitrary threshold of around half the local Alfvén speed, on this basis spikes occur every $\approx 19$ min on average filling $\approx 5$ per cent of the wind, a remarkably high fraction. The simulations of Uritsky et al. (2017) 


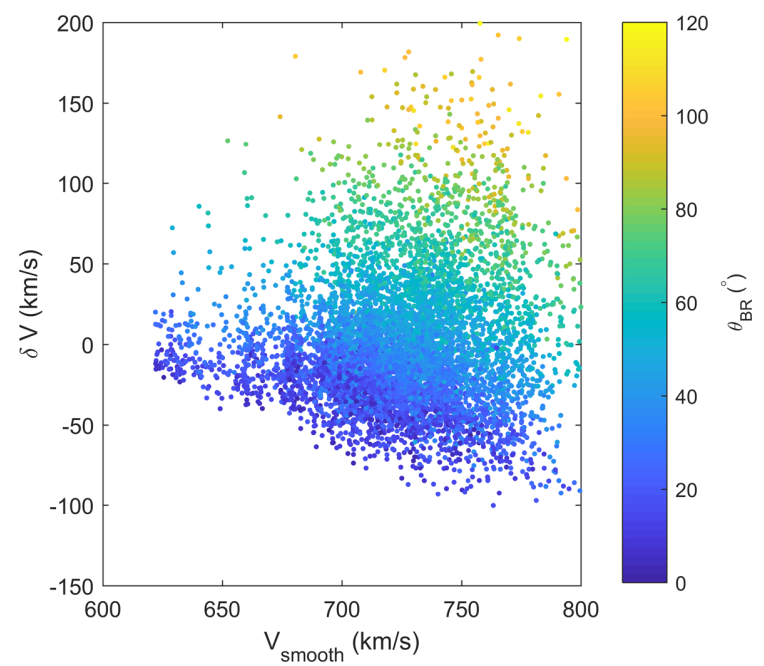

Figure 8. Deviations of the plasma speed from the $30 \mathrm{~min}$ smoothed mean. Colours of the dots represent angle of the local magnetic field to the radial.

suggest that the Alfvénic component of coronal jets tends to generate fine scale structure; it might be, therefore, that multiple in situ spikes are related to a single event closer to the Sun.

Preliminary examination of the detailed magnetic field structure associated with the spikes shows that many have helical substructure, but without any consistent signatures between them. Detailed comparison with simulation results might allow a better test of models of jet formation.

\subsection{Future missions}

The imminent launch of Parker Solar Probe and Solar Orbiter into the inner heliosphere will result in new measurements of the fine scale structure of the high-speed solar wind with the prospect of determining unambiguously the origin of the spikes reported here. These small scale structures raise their own measurement challenges for plasma instruments however, both at Solar Orbiter's perihelion of $\approx 60 R_{\mathrm{S}}$ and even more so for Parker Solar Probe at $9.8 R_{\mathrm{S}}$. The boundaries of these events appear to be sharp discontinuities which will pass the spacecraft on time-scales of the order of $0.1 \mathrm{~s}$ or less (Kasper et al. 2016); typical velocity changes are of the order of $2 V_{\mathrm{A}}$ radially and $V_{\mathrm{A}}$ transverse at distances sampled by Helios and where $\delta \boldsymbol{B} /|B| \approx 1$. The spike amplitude is not expected to increase significantly further Sunward, as it scales as $2 V_{\mathrm{A}}<\delta \boldsymbol{B} /|B|>$ and $\delta \boldsymbol{B} /|B|<1$ close to the Sun - however due the Alfvén speed profile $V_{\mathrm{A}} \approx 1 / R$, the predicted radial and transverse changes remain substantial also at $10 R_{\mathrm{S}}$. For example, taking typical values $V_{\mathrm{A}}=500 \mathrm{~km} \mathrm{~s}^{-1}, \delta \boldsymbol{B} /|B| \approx 0.2$, one gets velocity variations near $200 \mathrm{~km} \mathrm{~s}^{-1}$. Such large rapid changes can be challenging for ion detectors which operate in a 'search-track' duty cycle, if the changes take the core of the proton peak outside the field of view. In this case, detectors typically re-scan the entire velocity distribution, which could result in a slow measurement of a distribution function precisely when it is changing most rapidly.

\subsection{Turbulence analysis}

Some analysis methods of the field-aligned anisotropy of solar wind turbulence take advantage of changes in magnetic field direction and compares the spacecraft-frame power as the field angle varies with respect to the flow (e.g. Horbury, Forman \& Oughton 2008). Often implicit in such analysis is that the solar wind flow is radial and constant - and in particular does not vary systematically with field angle. The structures presented here have just such a systematic dependence. The velocity variations are of the order of the Alfvén speed: at $1 \mathrm{au}$ and beyond, this effect is small. At $0.3 \mathrm{au}$ it is more important and a preliminary analysis indicates that correcting for this effect affects power estimates by up to 15 per cent, but spectral indexes are not significantly affected. For Solar Orbiter and Parker Solar Probe, slightly more complicated analysis methods will probably be needed, to take into account the instantaneous velocity as well as field direction.

\section{CONCLUSIONS}

Sharp velocity increases appear ubiquitous in fast wind streams at $60 R_{\mathrm{S}}$. It is possible that they are simply part of an evolving population of waves carried from the corona by the solar wind. The discrete nature and very large amplitude of the events however, combined with their qualitative similarities to recent simulations of coronal jet signatures, raises the exciting possibility that they are the in situ signatures of these transient solar events. If so, this has implications for the energy budget of the solar wind, with jets contributing a non-trivial fraction of the total momentum and energy. It also makes available a new method of studying these jets and observationally distinguishing between models of their formation.

While the data used here are remarkable in their quality given their age, the imminent launch of Parker Solar Probe will provide data of much higher precision and cadence, far closer to the Sun, which will reveal any internal structure of these events; Solar Orbiter will then provide the solar context by which the link between solar and in situ transients will be clarified.

\section{ACKNOWLEDGEMENTS}

Work by T. S. Horbury and L. Matteini was supported by Science and Technology Facilities Council grant ST/K001051/1; David Stansby is supported by a studentship under Science and Technology Facilities Council grant ST/N504336/1. The authors gratefully acknowledge the provision of Helios data by U. Kiel and the original instrument teams.

\section{REFERENCES}

Bavassano B., Dobrowolny M., Mariani F., Ness N. F., 1982, J. Geophys. Res.-Space Phys., 87, 3617

Bruno R., , Carbone V., Sorriso-Valvo L., Bavassano B., 2003, J. Geophys. Res.-Space Phys., 108, 1130

Bruno R., Bavassano B., Villante U., 1985, J. Geophys. Res.-Space Phys., 90, 4373

Bruno R., Carbone V., 2013, Living Rev. Sol. Phys., 10, 7

Bruno R., Trenchi L., 2014, ApJ, 787, L24

Cranmer S. R., , Woolsey L. N., 2015, ApJ, 812, 71

Cranmer S. R., 2002, Space Sci. Rev., 101, 229

Cranmer S. R., Gibson S. E., Riley P., 2017, Space Sci. Rev., 212, 1345

Elliott H. A., , Henney C. J., McComas D. J., Smith C. W., Vasquez B. J., 2012, J. Geophys. Res.-Space Phys., 117, A09102

Gosling J. T., , Tian H., Phan T. D., 2011, ApJ, 737, L35

Hellinger P., Travnicek P. M., 2014, ApJ, 784, 5

Horbury T. S., Balogh A., 2001, J. Geophys. Res.-Space Phys., 106, 15929

Horbury T. S., Forman M., Oughton S., 2008, Phys. Rev. Lett., 101, 175005

Jackson B. V., Yu H. S., Buffington A., Hick P. P., 2014, ApJ, 793, 7

Karpen J. T., DeVore C. R., Antiochos S. K., Pariat E., 2017, ApJ, 834, 62 
Kasper J. C. et al., 2016, Space Sci. Rev., 204, 131

Marsch E., Muhlhauser K. H., Schwenn R., Rosenbauer H., Pilipp W., Neubauer F. M., 1982, J. Geophys. Res.-Space Phys., 87, 52

Matteini L., Horbury T. S., Neugebauer M., Goldstein B. E., 2014, Geophys. Res. Lett., 41, 259

Matteini L., Horbury T. S., Pantellini F., Velli M., Schwartz S. J., 2015, ApJ, 802,11

Matthaeus W. H., Goldstein M. L., 1986, Phys. Rev. Lett., 57, 495

Musmann G., Neubauer F. M., Maier A., Lammers E., 1975, Raumfahrtforschung, 19, 232

Neugebauer M., 2012, ApJ, 750, 50

Raouafi N. E. et al., 2016, Space Sci. Rev., 201, 1
Roberts M. A., Uritsky V. M., DeVore C., Karpen J. T., 2017, preprint(arXiv: 1710.10323)

Schwenn R., Rosenbauer H., Miggenrieder H., 1975, Raumfahrtforschung, 19,226

Thieme K., Schwenn R., Marsch E., 1989, Adv. Space Res., 9, 127

Tian H. et al., 2014, Science, 346, 1255711

Uritsky V. M., Roberts M. A., DeVore C. R., Karpen J. T., 2017, ApJ, 837, 123

Wyper P. F., Antiochos S. K., DeVore C. R., 2017, Nature, 544, 452

This paper has been typeset from a $\mathrm{T}_{\mathrm{E}} \mathrm{X} / \mathrm{L} \mathrm{AT} \mathrm{E} \mathrm{X}$ file prepared by the author. 